\title{
Improvement of the Vehicle License Plate Recognition System in the Environment of Rain and Fog
}

\author{
Zhun Wang ${ }^{1, a^{*}}$, Zhenyu Liü,b \\ ${ }^{1}$ Department of Information,Shenyang University of Technology,Shenyang,110870,China \\ ${ }^{2}$ Department of Information,Shenyang University of Technology,Shenyang,110870,China \\ aemail: zhunwang123456@163.com, bemail:liu_zhenyu0419@sina.com
}

\begin{abstract}
Keywords: License Plate Recognition; Gamma Correction; Double Color Space; Neural Network Recognition; Adaptive Fusion Algorithm

Abstract. License Plate Recognition (LPR) is one of the key technologies of the intelligence of communication management. A certain amount of difficulties to license plate recognition are caused by the environment of rain and fog. License plate recognition system for this kind of environment is studied in this paper, based on the theory of digital image processing, computer vision and pattern recognition technology. In order to improve the ability to identify the license plate, Gamma correction algorithm and the denoising algorithm of color image are added to license plate locating method based on color. In the pre-processing of the segmentation method based on connected area detection, a kind of double color space binarization method is proposed by the article. Finally a kind of adaptive fusion algorithm based on BP, RBF, GRNN neural network is proposed to finish the recognition for license plate character. Experiment shows that the method adopted by this system applied in the bad environment of rain and fog achieves good recognition effect.
\end{abstract}

\section{Introduction}

Along with the social life rhythm speeding up, the vehicle popularization has become inevitable trend of social development. In a large vehicle management system, license plate recognition technology is a key technology in intelligent traffic management system, which is widely applied to highway, intelligent building entrances, airport, parking management and so on. In a license plate recognition system, due to the influence of the weather condition, especially rain and fog, there are many factors that can affect the recognition results of the system, such as background complexity increasing, light intensity weakening, license plate stained and so on. The image processing part of the system and license plate recognition algorithm need to adapt to the condition mentioned above. In view of the license plate recognition of the special conditions, targeted research and improvement on license plate recognition from three phases of license plate location, license plate segmentation and license plate recognition by this article.

\section{License Plate Positioning}

There are many kinds of algorithms of license plate location., such as frequency domain analysis method based on DFT transform, location identification method based on neural network, license plate location method based on Hausdoff and so on. The above methods can extract license plate images under certain conditions, but often fail to have an ideal effect of positioning in weather conditions of rain and fog, where there are many interference, such as blurred images, the change of light and soiled license plate. They don't have very strong adaptability to the environment and the characteristics of domestic license.In this paper, a license plate locating method based on color and texture have been adopted, according to the characteristics of domestic license plate.

License plate locating is completely splitting the license plate area image from the background of a vehicle image, whose basic idea is to finish extraction of license plate area image according to the different characteristics of license plate region and background region. But vehicle images are blurred on the whole in the weather of rain and fog, and their pixels are low, making the difference and the border of license plate region and background region weaken and fuzzy, so as to increase the difficulty of license plate locating. In order to weaken the disturbance of environment of rain and fog 
to license plate image positioning, and to increase the contrast of license plate region and background region,Gamma algorithm and Gaussian denoising algorithm of color image are added in the basis positioning algorithm. The process of positioning is shown in Fig. 1.

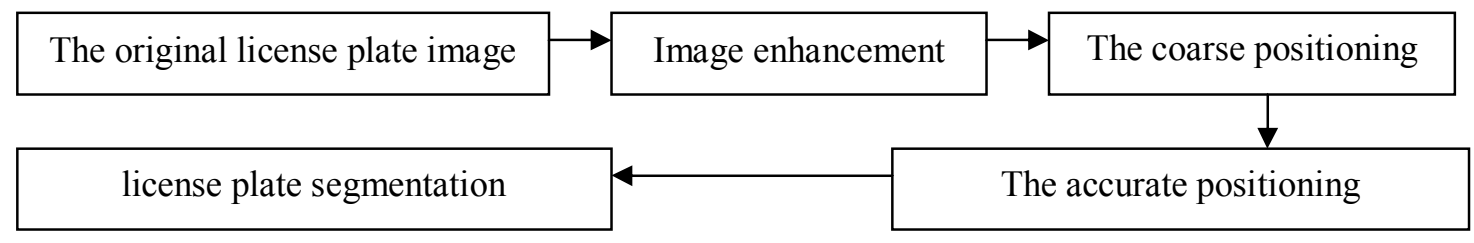

Fig. 1 Flow chart of positioning

Gamma correction is a kind of parameter values, which is used to express the nonlinear characteristics of the CRT (cathode ray tube) display. In addition, the sense of human visual system to the brightness or RGB signals of three colors follow the law of logarithmic, which is not a linear relationship. Gamma correction method is introduced as a transfer function in order to overcome the nonlinearity. In general, when Gamma correction value is greater than 1, highlight part of the image is compressed and the shade part is extended; when the value is less than 1, the highlight part is extended and the shade part is compressed.

Under the interference of the weather of rain and fog, image is more fuzzy compared with the normal image, whose color is relatively shallow, which is not conducive to license plate positioning based on color and texture. In the system, the parameter of Gamma correction is set to 1.2, which darken the image on the whole and deepen the color of blue license plate compared with the color of background part,thus in favor of the extraction of license plate images. Contrast of before and after the image enhancement of license plate images is shown in Fig. 2.

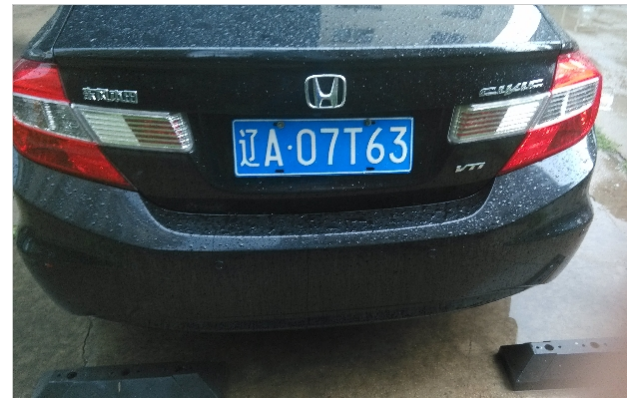

(a) before image enhancement

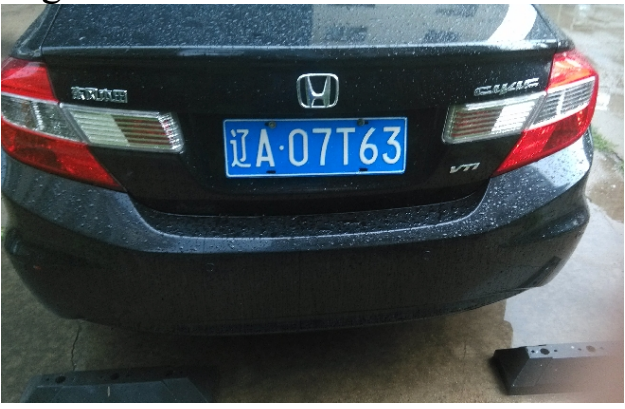

(b) after image enhancement

Fig.2. contrast of before and after the image enhancement of license plate image

After image enhancement, the image intensified by Gamma algorithm is transformed from the RGB color space to the HSV color space. Then the tonal component matrix and the saturation component matrix of the image are input into filter in order to remove their Gaussian noise. The size of denoising template is taken for [3,3], which can meet the requirements of two aspects of denoising performance and image resolution. After the binarization of the tonal component and the saturation component of the image, we AND them to each other. Then the coarse positioning is finished by a series of morphological processing, including opening operation, closing operation and convex hull operation. In view of the factor of the wear and tear of license plates and the effect of light in the weather of rain and fog, the threshold of binarization of the saturation component matrix is set as 0.6. After coarse positioning in color space, the position of connected domain whose area is overlarge is removed, if there exists, by connected components detection, in order to rule out the situation that vehicle appearance is blue. Finally accurate positioning of license plate image is finished by testing the position of the connected domain whose area is largest in this image.

Affected by the shooting angle, the license plate image may be tilted at an angle. After license plate positioning, the angle of inclination of the plate is determined by testing the angle of $\mathrm{x}$ axis and the axis of the ellipse that has the same standard second-order central moment as the plate region. Finally the license plate image is rotated at the tested angle for tilt correction. 


\section{Character Segmentation}

A character segmentation method based on double color space and connected component detection is presented by this article. Under the weather of rain and fog, the fuzziness of the boundary of license plate characters and blue background can be caused by fog and water vapor between shot and license plate. And a certain degree of distortion of character area may be made by refraction effect of light presented by water drops attached on license plate. In addition, segmentation results are more likely affected by the dust and dirt of license plate itself in the environmental conditions of rain days. Due to the influence of the above in the weather of rain and fog, some algorithms that can achieve correct segmentation in fine weather, where guidelines of Identifying characters and background may fail in such bad weather, lead to segmentation fault. To solve this problem,we must as far as possible reduce the influence of rain and fog for license plate images before segmentation. Therefore improvement of pre-processing of segmentation algorithm is necessary.

At first, the license plate image is transformed from the RGB color space to the HSV color space, and then we binary the saturation component. The threshold value of binarization is set by automatically computing with a statistical method, rather than directly determined as a fixed value or average, like most of the license plate recognition systems. Threshold calculation formula is:

$$
t h_{2}=m_{2}+0.2 \times s_{2}
$$

In the formula, $\mathrm{th}_{1}$ stands for the threshold; $\mathrm{m}_{1}$ is the mean value of the saturation component matrix, and $\mathrm{s}_{1}$ is the mean square error value of the saturation component matrix.

The mean square error value is introduced into threshold calculation formula, in order to minimize the interference of environment, making the character outline more clearly. It is proved that the method of threshold determination has an obvious improvement effect on the process of segmentation and recognition under the weather of rain and fog in the experiment.

Then we binary the red component of license plate image in the RGB color space, for blue background of license plate and white characters reach the maximum degree of differentiation in the red component. Threshold is determined by computing with a statistical method likewise. Threshold calculation formula is:

$$
t h_{2}=m_{2}+0.2 \times s_{2}
$$

In the formula, $t_{2}$ stands for the threshold; $\mathrm{m}_{2}$ is the mean value of the red component matrix, and $\mathrm{s}_{2}$ is the mean square error value of the red component matrix.

In the environment of rain and fog, white characters are more likely to be affected by dust and dirt, which brings about the change of their color component. Therefore the mean square error is multiplied by 0.2 , in order to adapt to the change caused by dust and dirt on white characters and increase the accuracy of the binarization.

Then we AND the two results of the binarization respective in two color space to each other.

The noise of threshold image caused by water drops, dust and dirt is further eliminated by median filtering. The intermediate region of license plate is extracted with priori knowledge, and then, in order to eliminate the influence of rivet area, the region outside the upper and lower boundary of the characters is removed, by detection of area and shape of connected regions. License plate characters, in addition to the Chinese character, are segmented by using sorting of area and centroid as well as segmentation method based on connected component detection; the Chinese character is segmented out by priori knowledge. Finally all characters are scaled to a unified standard size.

\section{License Plate Character Recognition}

There are several frequently used character recognition method: neural network pattern recognition, statistic pattern recognition, structural pattern recognition, fuzzy pattern recognition, and so on. Neural network pattern recognition can deal with some problems where environmental information is very complex, and inference rules are not clear, and background knowledge is not clear. This method allows images have relatively large image defect and distortion, and has certain characteristics of 
intelligent processing. In the environmental conditions of the fog and rain, the license plate images tend to have larger defects and distortion, so neural networks are adopted by this article for character recognition. But each neural network has its respective adaptability and limitation. For example, as a most widely used neural network, BP neural network has the problem of easily falling into local minimum. In order to achieve high recognition rate in the complex environment, this paper proposed an adaptive fusion algorithm based on BP neural network, RBF neural network, and GRNN neural network to realize character recognition. This algorithm can process characters on the license plate images with large noise by intelligent processing, and can also avoid some situations that a single neural network fail to identify characters or has identification errors, thus further improve the accuracy of license plate character recognition.

This paper adopts characters of the grid feature extraction approach, which directly inputs elements of the whole gray scale image into neural network, getting rid of the feature extraction and enhancing the anti-jamming performance of the network. This approach is conductive to the network to keep high recognition rate.

\section{BP neural network}

The BP neural network is a kind of inverse learning algorithm of multi-layer network. Its network model is composed of neuron nodes of several layers, shown as Fig.3, including the input layer, hidden layers, and the output layer. Each layer is composed of multiple neurons.

input layer hidden layer hidden layer output layer

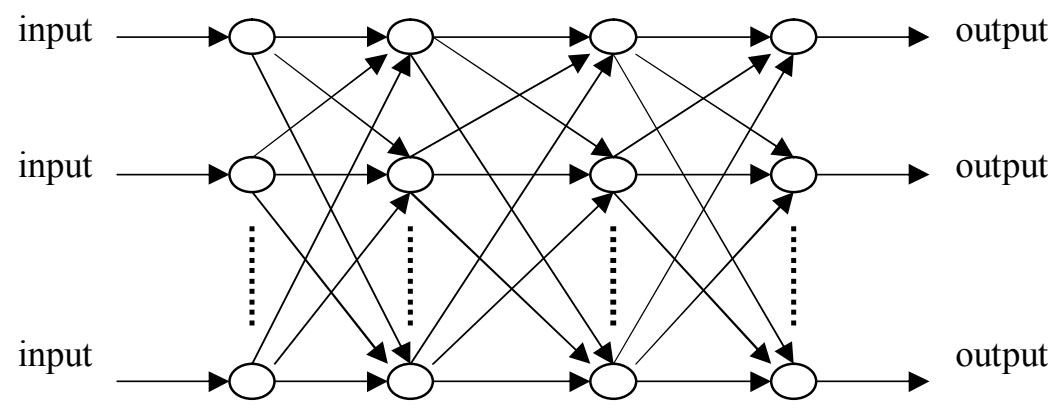

Fig.3 BP network structure

$\mathrm{y}_{\mathrm{i}}{ }^{(\mathrm{k}-1}$ is set as the input to the ith neuron of the $\mathrm{k}-1$ th layer, and the $\mathrm{y}_{\mathrm{i}}{ }^{\mathrm{k})}$ is set as the output. The relationship between the input and output is:

$$
y_{j}^{(k)}=f\left(\sum_{i=1}^{N_{k-1}} W_{i j}^{(k-1)} y_{i}^{(k-1)}-\theta_{j}^{k}\right)
$$

Before the application of BP neural network, it need to be trained self-learning. The process of learning algorithm of BP neural network is:

(1) The initial values are set(connection weights $w$ and threshold value $\theta$ );

(2) A set of training data is input into the network;

(3) Forward calculation from the input to the output;

$$
y_{j}^{(m)}=f\left(s_{j}^{(m)}\right)=f\left(\sum_{i} w_{j i}^{(m)} \times y_{i}^{(m-1)}-\theta_{j}^{(m)}\right)
$$

(4) The errors of output data of each neuron in the output layer are calculated;

$$
\delta_{j}^{(m)}=f\left(s_{j}^{(m)}\right)=f^{\prime}\left(\sum_{i} w_{j i}^{m} \times y_{i}^{(m-10}-\theta_{j}^{(m)}\right)
$$

(5) The errors of each neuron in the last layer are calculated;

$$
\delta_{j}^{(m)}=f^{\prime}\left(s_{j}^{(m-1)}\right) \sum_{i} w_{j i}^{(m)} \boldsymbol{\delta}_{i}^{(m)}
$$

(6) The connection weights are modified; 


$$
\begin{aligned}
\Delta w_{j i}^{(m)} & =\eta \delta_{j}^{(m)} y_{i}^{(m-1)} \\
w_{j i}(t+1) & =w_{j i}(t)+\Delta w_{j i}(t)
\end{aligned}
$$

(7) Return to step 2 until the network convergence, that is, $\mathrm{E}<\mathrm{e}_{0 .}$

S-type function is the excitation function of the BP neural network. When constructing the BP neural network in the experimental system, set the learning rate of training as 0.15 , the maximum number of iteration as 12000 , the minimum mean square error as 0.001 , and the factor of momentum as 0 .

\section{BRF neural network}

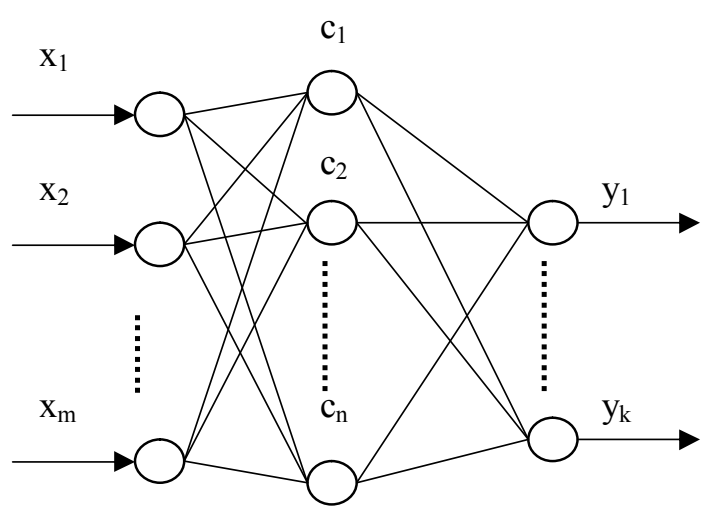

Fig.4 RBF network structure

Multivariate interpolation of radial basis function(BRF) network, belongs to the type of feed-forward neural networks, which can approximate any continuous function with arbitrary precision so as to be particularly suited to solve classification problems. This network is a kind of feed-forward neural networks of excellent performance, with compact topological structure, fast convergence speed, and structure parameters which can achieve separately learning., and fundamentally solve the problem of local optimal of BP network. RBF network and fuzzy logic can complement each other well, thus improve the abilities of learning and generalization of neural network. Its structure is shown as Fig.4.

As is widely used as the radial basis function of network, Gaussian function is set as the radial basis function of the RBF neural network in this article. Its formula is:

$$
R_{i}(P)=\exp \left[-\frac{\left\|P-C_{i}\right\|^{2}}{\sigma_{i}^{2}}\right]
$$

The self-organize algorithm is adopted by this article to find the location of RBF centers, which is one of the many kinds of learning algorithm of RBF neural network. This algorithm includes two stages, supervised learning and self-organized learning. Gaussian function is radially symmetric, and is proportional to the response according to the output of the hidden layer of RBF neural network. When the input is the same as the radial distance of the center of basis function, their outputs of the nodes of hidden layer are the same. And the center of hidden layer nodes $C_{i}$ and the radius of implicit function $\delta_{\mathrm{j}}$ are needed to worked out.

The center of basis function of the RBF network is worked out by k-mean clustering algorithm, whose process is:

(1) The center of clustering is initialized, that is, randomly select I different samples from training samples are randomly selected as the initial center $\mathrm{t}_{\mathrm{i}}(0)(\mathrm{i}=1,2, \ldots, \mathrm{I})$, according to the experience. And the number of iteration steps $\mathrm{n}$ as 0 ;

(2) Training samples $X_{k}$ are randomly input into the network;

(3) The center which is nearest to training samples $X_{k}$ is found, that is, the $i\left(X_{k}\right)$ that comply with the following formula is found:

$$
i\left(X_{k}\right)=\arg \min \left\|X_{k}-t_{i}(n)\right\|, i=1,2, \ldots I
$$

In the formula, $\mathrm{t}(\mathrm{n})$ is the nth center at the nth iteration.

(4) The center is adjusted by the following formula: 


$$
t_{i}(n+1)=\left\{\begin{array}{c}
t_{i}(n)+\eta\left(X_{k}-t_{i}(n)\right), \text { 当 } i=1,2 \ldots, I \\
t_{i}(n)
\end{array}\right.
$$

(5)The judgment is made that the process of learning all the training samples with the results of changeless distribution have been finished or not. If yes, the process of learning is ended up; otherwise, $n=n+1$, and return to (2).

The calculated $\mathrm{t}(1,2, \ldots \mathrm{I})$ are the final center of basis function.

The fundamental principle of determining the radius of implicit function is to make the sum of receiving domains of all the hidden units covers the whole sample space. After the sample classification, radius of implicit function is calculated according to K-nearest algorithm:

$$
\delta_{j}=\sqrt{\frac{1}{K} \sum_{k=1}^{K}\left\|x(k)-C_{j}\right\|^{2}}
$$

The RBF neural network is trained, by using linear least square method, and then the connection weights between the hidden layer and the output layer are worked out. The number of neurons in the hidden layer is set as 25 , and the largest number of neurons is set as 1400 . At first sample mode counter $\mathrm{p}$ and training times $\mathrm{q}$ are set as 1 . The error $\mathrm{E}$ is initialized as 0 , and the error threshold of the experiment $\mathrm{E}_{\min }$ as 0.05 . Then the initial learning rate is set as 0.6 . The network's general error is:

$$
E=\sqrt{\frac{1}{P} \sum_{p=1}^{P}\left(E^{p}\right)^{2}}
$$

\section{GRNN neural network}

Generalized regression neural network(GRNN) is a kind of new neural network proposed by American scholar Donald F.Specht in 1991, one kind of radial basis function(RBF) neural network. Set on the basis of $\mathrm{P} \sim \mathrm{n}$ non-parametric estmation, the network can estimate continuous variables and converges in the basic regression surface. Set on the basis of mathematical statistics, GRNN neural network is also the extended form of probabilistic neural network(PNN). GRNN neural network can solve nonlinear problems well, with strong ability of nonlinear mapping and high degree of fault tolerance and robustness. The structure of GRNN network is shown as Fig.5.

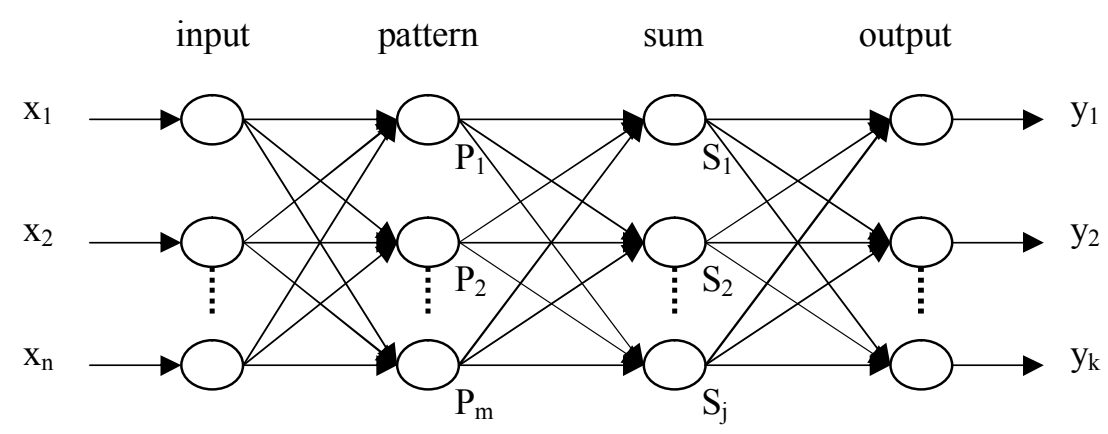

Fig.5 GRNN network structure

GRNN neural network is mainly composed of four parts of structure: input layer, model layer, summation layer, and output layer. With clear meaning of possibility and completely specified structure and connection weights, GRNN neural network overcomes the difficulties on design and train of general RBF network, and though lack of sample data, GRNN network can also achieve excellent predicting results, with faster train speed and stronger nonlinear mapping capability. The network can finish training within a moment with a high fitting precision. The expression of the output of GRNN network is: 


$$
\begin{gathered}
Y(X)=\frac{\sum_{i=1}^{n} Y_{i} \exp \left(-\frac{D_{i}^{2}}{2 \sigma^{2}}\right)}{\sum_{i=1}^{n} \exp \left(-\frac{D_{i}^{2}}{2 \sigma^{2}}\right)} \\
D_{i}^{2}=\left[\left(X-X_{i}\right)^{T}\left(X-X_{i}\right)\right]
\end{gathered}
$$

In the formula, $X_{i}$ and $Y_{i}(i=1,2, \ldots n)$ are the inputs and outputs of the ith sample, respectively. And $n$ is the number of samples. $X$ stands for the input vector, and $Y(X)$ stands for the output of the network when the input is X. $\sigma$ stands for the width of sensing field. Different training effect is realized by adjusting the value of $\sigma$. Newgrnn is the constructor function of GRNN neural network, whose format is:

$$
[n e t, t r]=\operatorname{newgrnn}(P, T, S P R E A D)
$$

SPREAD, the smoothing parameter of basis function, remains the default 1.0.

On the basis of three methods of character recognition by neural network, adaptive fusion algorithm based on the three neural network is proposed by this article.

\section{adaptive fusion algorithm}

The three neural networks above are trained by a large number of image files, producing three trained neural networks respectively named ym_BP.dat, ym_RBF.dat and ym_GRNN.dat. Three groups of recognition results are obtained by inputting the image after positioning and segmentation to the three trained networks in the system. The information fusion follows the principle as follows: If results of three three groups are the same, the final result is determined as the only result; If results of only two of the three groups are the same, the final result is determined as the same result of the two groups; If results of three groups are different, then the final result is determined as the result of GRNN neural network which has the highest accuracy in the three networks. The logic diagram of recognition is shown as follows:

$$
\left\{\begin{array}{l}
A \cap A \cap A \Rightarrow A \\
A \cap A \cap B \Rightarrow A \\
A \cap B \cap C \Rightarrow X_{R G N N}
\end{array}\right.
$$

\section{Test results}

The algorithms of all modules have been realized through programming in Matlab., and the experiment effect is shown as Figure 6. 


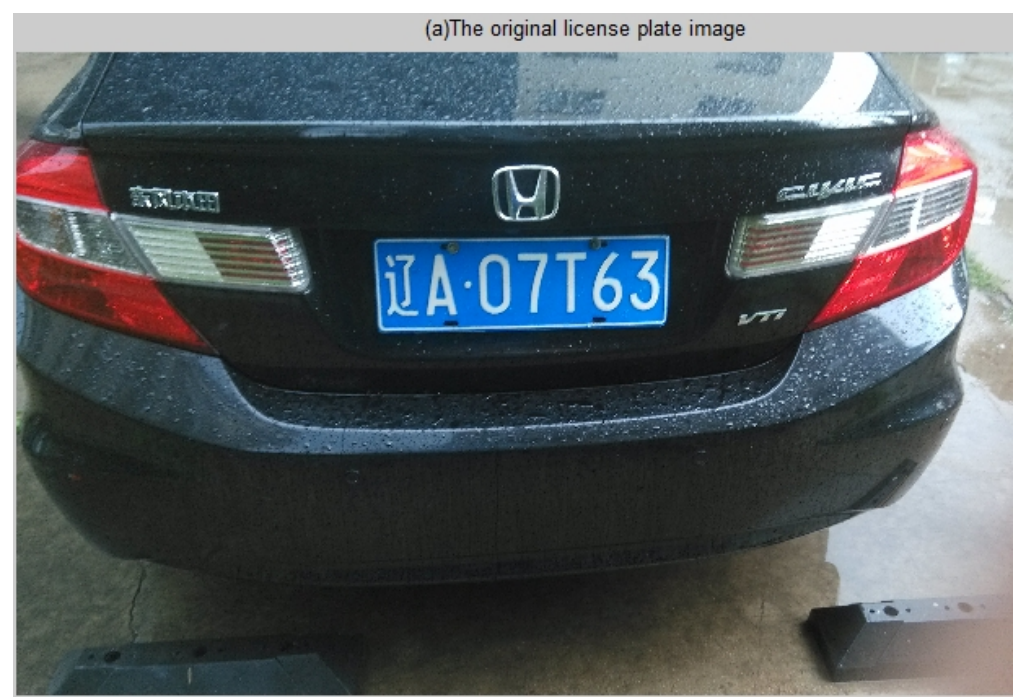

(a) The original license plate image

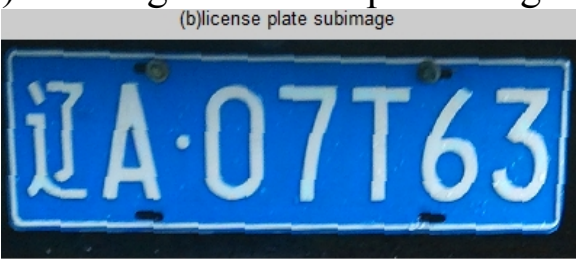

(b) License plate image

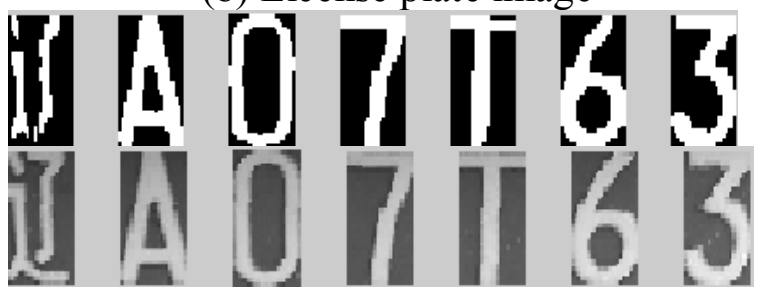

(c) License plate characters after segmentation

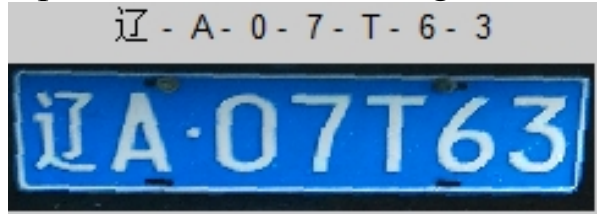

(d) The result of license plate recognition

Fig. 6 The result of experiment

805 license plate pictures in different period of time and different angle of inclination are collected by shooting with digital camera and surfing on the internet, in which there are 377 license plate images shooting in the weather conditions of rain and fog. All 805 license plate images are composed of 10 figures, 24 English letters and Chinese characters including Gui, Su, Liao, Jing, Min ,Yue ,Qiong, Ji, E, Xiang, Ji, Gan, Meng, Ning, Qing, Lu, Jin, Shan, Hu, Chuan, Jin, Xin, Zhe, $\mathrm{Yu}$. Typical license characters are selected from the image library to input the three neural networks after segmentation for training of networks, until the three networks achieve their states of convergence. Then the overall performance of this license plate recognition is tested.

All the 377 license plate images in the weather of rain and fog in the image library are input to the experimental system to test the system performance, in which there are 369 license plate images of correct recognition, and 8 of error recognition. The rezults of the experiment are shown in Table 1 . Experimental results shows that the license plate recognition system we build for the weather of rain and fog have achieved $97.8 \%$ of high recognition rate. The correct recognition' means that no error character exists in the result of recognition. In order to further study its performance, this system is compared with another license plate recognition system with no improvement. It is found that the effect of the segmentation achieves big improvement, and the recognition algorithm achieves higher character recognition rate in our system. But as the price of improvement of recognition rate, the speed of recognition has been a little slower. And it is found in the results that 4 highly reflective 
photos shot at night have been not correctly recognized, accounting for half of the number of error recognition images. So results are not satisfactory when highly reflective images are processed by the system, which is the defect of this system.

Table 1 License plate recognition result statistics

\begin{tabular}{cccc}
\hline System & Total & $\begin{array}{c}\text { Number of correct } \\
\text { recognition }\end{array}$ & recognition accuracy \\
\hline $\begin{array}{c}\text { The improved } \\
\text { license plate } \\
\text { recognition }\end{array}$ & 370 & 362 & $97.83 \%$ \\
$\begin{array}{c}\text { Contrasting } \\
\text { system }\end{array}$ & 370 & 298 & $80.54 \%$ \\
\hline
\end{tabular}

\section{Conclusion}

In view of the difficulties caused by the weather of rain and fog to license plate recognition, some improvements and innovation are made in image enhancement, license plate positioning, pre-processing of license plate segmentation and fusion of multiple neural networks. It is proved that this system of license plate recognition has achieved good recognition performance through the experiment.

\section{Acknowledgements}

[1] Industrial project of Shenyang technology bureau, research and development of multi-channel video enhancement system, project number: F14-073-2-00

[2] Education department of liaoning province, project number:2014041

\section{References}

[1] Liangliang He. Study and Realization of A Kind of License Plate Recognition Technology in Chinese [D]. South China University of Technology, 2014.

[2] Zhifan Feng. Study of License Plate Recognition Based on Graphic Processing and BP neural network in Chinese[D]. Wuhan University of Science and Technology, 2011.

DOI:10.7666/d.y1943836.

[3] Weiwei Chen. System of License Plate Recognition and Information Management Based on Neural Network in Chinese[D]. Wuhan University of Technology, 2010. DOI:10.7666/d.y1680897.

[4] Dongjian He, Nan Geng, Yikuan Zhang. Digital Image Processing. Xi'an: Xi'an Electronic Sience \&Technology University Press, 2008

[5] Leilei Zhang. Study of License Plate Recognition Based on Radial Basis Function in Chinese[D]. Xi'an University of Science and Technology, 2013

[6] Li Wang, Xiaohua Wang. Application of Neural Network in License Plate Recognition in Chinese [J]. Microcomputer and Its Applications[J],2011, 30(5):38-40 DOI:1674-7720 (2011) 05-0038-03

[7] Xiaochun Zhang, Kaichun Ren. Study of License Plate Recognition Based on RBF Neural Network in Chinese[J]. Chinese Journal of Automotive Engineering, 2012, 2(2):147-151 DOI: 10. 3969/j.issn.2095-1469.2012.02.010

[8] Fuqiang Xu, Tingting Zheng. Function Approximation Based on Generalized Regression Neural Network(GRNN) in Chinese[J]. Journal of Chaohu University,2010,12(6):11-16 DOI:1672-2868. (2010)06-0011-06

[9] Yan Zhang, Anhu Ren. Study of Multiple Features and License Plate Recognition System Based on BP Neural Network in Chinese[J]. Science Technology and Engineering, 2012,12(22) DOI: 16711815.(2012)22-5645-04

[10] Zhibang Xu, Xiaoying Shi. Design and Realization of Automatic License Plate Recognition System Based on RBF Neural Network in Chinese[J]. Journal of Nanchang

University,2009,31(2):147-150 DOI:1006-0456.(2009)02-0147-04 
[11] Defeng Zhang. Application Design of Neural Network in MATLAB in Chinese[M]. Beijing: China Mechine Press,2012:136-186 